\title{
Etes-vous bien conseillé(e)?
}

FMH Fiduciaire Services - principale société fiduciaire pour le corps médical

Vous recherchez de l'aide pour créer ou gérer un cabinet médical ou encore pour un cas de succession? Quel que soit votre besoin - élaboration d'un plan d'affaires, comptabilité, clôture de l'exercice, déclaration d'impôts ou révision des comptes - nos experts fiduciaires vous apporteront des solutions sur mesure. Nous sommes bien implantés dans les trois régions linguistiques, et parfaitement au fait de la législation cantonale et des dispositions légales spécifiques à votre profession.

\section{Nous vous proposons un large éventail de prestations de services}

\section{Finances et comptabilité}

- Plan d'affaires, plan financier

- Gestion de la trésorerie

- Comptabilité

- Compte des investissements

- Tenue des comptes créditeurs et débiteurs avec gestion des relances
- Possibilités de financement

- Aide à l'établissement et à la clôture des comptes

- Administration du personnel et comptabilité des salaires

- Etablissement des décomptes d'assurances sociales

Analyses

- Analyse de l'état financier et du résultat en cas de problèmes financiers

- Interprétation du «Miroir du cabinet» (TrustCenter)

Fiscalité

- Etablissement des déclarations d'impôts

- Conseil en matière d'impôts et planification fiscale stratégique
- Conseil en matière de TVA

- Etablissement du décompte de TVA

Faites confiance à notre vaste et solide réseau de spécialistes!

\section{Talon-réponse}

Prénom / Nom

Adresse

NPA / Lieu

Date de naissance

Téléphone privé / cabinet

Atteignable le plus facilement (heure)

Je suis intéressé(e) par les prestations de FMH Fiduciaire Services.

Veuillez me contacter. 